\title{
Anxiety and Depression in Patients with Chronic Obstructive Pulmonary Disease in China: Results from the China Pulmonary Health $[\mathrm{CPH}]$ Study
}

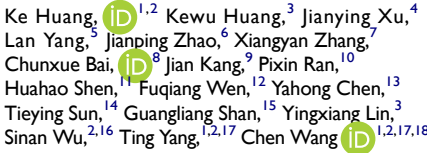

'Department of Pulmonary and Critical Care Medicine, Center of Respiratory Medicine, China-Japan Friendship Hospital, Beijing, People's Republic of China

${ }^{2}$ Department of Pulmonary and Critical Care Medicine, National Clinical Research Center for Respiratory Diseases, Beijing, People's Republic of China; ${ }^{3}$ Beijing Key Laboratory of Respiratory and Pulmonary Circulation Disorders, Department of Pulmonary and Critical Care Medicine, Beijing Chao-Yang Hospital, Capital Medical University, Beijing, People's Republic of China; ${ }^{4}$ Departy. Befing People's Repulic of China; Shax Day Hospial, Tayun, Peoples Republic of Shanxi Dayi Hospital, Taiyuan, People's Republic of China; ${ }^{5}$ Department of Pulmonary and Critical Care Medicine, First Affiliated Hospital of Xi'an Jiaotong University, Xi'an, People's Republic of China; ${ }^{6}$ Department of Pulmonary and Critical Care Medicine, Tongji Hospital, Tongji Medical College, Huazhong University of Science and Technology, Wuhan, People's Republic of China; 'Department of Pulmonary and Critical Care Medicine, Guizhou Provincial People's Hospital, Guiyang, People's Republic of China; 'Department of Pulmonary and Critical Care Medicine, Zhongshan Hospital, Fudan University, Shanghai, People's Republic of China; ${ }^{9}$ Department of Pulmonary and Critical Care Medicine, Firs Hospital of China Medical University, Shenyang, People's Republic of China; ${ }^{10}$ State Key Laboratory of Respiratory Disease, National Clinical Research Center for Respiratory Diseases, Guangzhou Institute of Respiratory Diseases, First Affiliated Hospital, Guangzhou Medical University, Guangzhou, People's Republic of China; ' Department of Hospital of Zhejiang University, School of Medicine, Hospital of Zhejiang University, School of Medicine,
Hangzhou, People's Republic of China; ${ }^{2}$ State Key Laboratory of Biotherapy of China and Department of Respiratory and Critical Care Medicine, West China Hospital of Sichuan University, Chengdu, People's Republic of China;

${ }^{13}$ Department of Respiratory and Critical Care Medicine Peking University Third Hospital, Beijing, People's Republic of China; ${ }^{14}$ Department of Respiratory and Critical Care Medicine, Beijing Hospital, Beijing, People's Republic of China; ${ }^{15}$ Institute of Basic Medical Sciences, Chinese Academy of Medical Sciences, School of Basic Medicine, Peking Union Medical College, Beijing, People's Republic of China;

${ }^{16}$ Institute of Clinical Medical Sciences, Center of Respiratory Medicine, China-Japan Friendship Hospital, Beijing, People's Republic of China; Department of Respiratory Medicine, Capital Medical University, Beijing, People's Republic of China; ${ }^{18}$ Institute of Respiratory Medicine Chinese Academy of Medical Sciences, Beijing, People's Republic of China

Correspondence: Chen Wang; Ting Yang Department of Pulmonary and Critical Care Medicine, Center of Respiratory Medicine, China-Japan Friendship Hospital, Chaoyang District, Beijing, 100029, People's Republic of China

Tel +86 I0 65I05565; +86 I0 84206276

Email cyh-birm@263.net;

dryangting@qq.com
Purpose: Anxiety and depression are often underdiagnosed and affect the prognosis of patients with chronic obstructive pulmonary disease (COPD). We analyzed data from the China Pulmonary Health (CPH) study to assess the prevalence of anxiety and depression in COPD patients and their relationship with disease severity.

Patients and Methods: A total of 57,779 subjects aged 20 years or older were recruited in the CPH study. All participants were assessed using a standard questionnaire and underwent pulmonary function tests before and after the use of a bronchodilator in local health centers. The Hospital Anxiety and Depression Scale (HADS) questionnaire with a cutoff score of 8 was used to define anxiety and depression. The prevalence of anxiety and depression in patients of COPD were investigated. Multivariate logistic regression was used to investigate the effects of COPD and lung function on anxiety and depression.

Results: A total of 49,053 participants (20,661 men and 28,392 women) completed the questionnaire with reliable post-bronchodilator pulmonary function test results and were included in the final analysis, of which 4686 (9.55\%) were diagnosed with COPD. Of the patients with COPD, $10.79 \%$ had anxiety, $13.65 \%$ had depression, and $7.08 \%$ had anxiety and depression concomitantly. In the multivariate logistic regression analysis, COPD was not significantly associated with anxiety, depression, or both. After adjusting for confounders in model 1, patients in the GOLD III-IV group had a significantly higher risk of anxiety, depression, and their coincidence. After further adjusting for respiratory symptoms in model 2, lung function impairment in the GOLD III-IV group was only significantly associated with a higher risk of depression or at least one of anxiety and depression.

Conclusion: Anxiety and depression are prevalent in patients with COPD in China. More severe lung function impairment is significantly associated with a higher risk of depression. Keywords: anxiety, COPD, depression, Hospital Anxiety and Depression Scale, HADS

\section{Introduction}

Chronic obstructive pulmonary disease (COPD) is a common chronic respiratory disease affecting approximately 99.9 million Chinese people, according to the China Pulmonary Health $(\mathrm{CPH})$ study, ${ }^{1}$ which is the largest survey evaluating the burden of COPD in China. COPD is the third leading cause of death and the fourth leading cause of years of life lost in China. ${ }^{2}$ Comorbidities, including anxiety and depression, are common in COPD patients and have a significant impact on prognosis. ${ }^{3,4}$ Anxiety and depression are common mental disorders and a recent survey reported that the lifetime prevalence of anxiety and depression disorders 
were $7.6 \%$ and $6.8 \%$, respectively, in China. ${ }^{5}$ Previous studies found that nearly one in four COPD patients suffered from depressive symptoms and up to $40 \%$ of COPD patients have clinical anxiety, although they are often undiagnosed. $^{6-9}$ Patients with anxiety and depression have impaired prognosis in terms of health-related quality of life, healthcare utilization, and mortality. ${ }^{10,11}$ Our previous study also showed that depression increases the risk of COPD exacerbations and hospitalizations. ${ }^{12}$ Many factors contribute to the development of anxiety and depression in COPD, but strangely, disease severity does not affect the level of anxiety and depression. ${ }^{13}$

Since previous studies used different tools to define anxiety and depression and were conducted in different settings, mostly in hospitals or primary care clinics, it is difficult to reach a consensus about their prevalence, especially among COPD patients from the general population. To our knowledge, there is no national survey evaluating the prevalence of anxiety and depression in COPD patients from the general population. In the present study, we used data from the CPH study to evaluate the prevalence of anxiety and depression among COPD patients and their relationship with disease severity.

\section{Patients and Methods}

\section{Study Population}

We used data from the previously published $\mathrm{CPH}$ study. ${ }^{1,14,15}$ Briefly, the CPH study is a national crosssectional study, including 57,779 Chinese adults aged 20 years or older, which was sampled using a multistage stratified cluster sampling procedure in 10 provinces across the country, including 80 urban sites and 80 rural sites. A standardized questionnaire, including information on sociodemographic status, medical history, smoking history, respiratory symptoms, anxiety, and depression, was administered to participants by trained health workers. Spirometry was performed by trained health workers using a standardized protocol for all participants. The Hospital Anxiety and Depression Scale (HADS) questionnaire $^{16,17}$ was used to evaluate anxiety and depression.

This study was conducted in accordance with the Declaration of Helsinki. The study protocol was approved by the Ethics Review Committees of the Capital Medical University (Beijing, China) and all other participating centers. Written informed consent was obtained from all participants.

\section{Procedures}

Lung function test procedures and definitions of COPD, respiratory symptoms, smoking status, and smoking exposure have been reported previously. ${ }^{1}$ Post-bronchodilator forced expiratory volume in $1 \mathrm{~s}\left(\mathrm{FEV}_{1}\right)$ and forced vital capacity (FVC) were measured using standardized spirometry. The post-bronchodilator $\mathrm{FEV}_{1} / \mathrm{FVC}$ ratio was used to define COPD, and the ratio of observed to predicted $\mathrm{FEV}_{1}$ ( $\mathrm{FEV}_{1} \%$ pred) was used to stage disease severity according to the 2017 Global Initiative for Chronic Obstructive Lung Disease (GOLD). Marital status and family income were included in the analysis. Comorbidities, including cardiovascular diseases (coronary heart disease, stroke, and hypertension), malignant tumors, and diabetes mellitus, were also evaluated.

Anxiety and depression were assessed using the HADS questionnaire, which has been validated as a tool for screening cases of anxiety and depression and evaluating symptom severity in general populations and patients with chronic diseases, including COPD. ${ }^{16}$ The Chinese HADS has been validated in the Chinese population. ${ }^{17}$ The HADS questionnaire has 14 items, 7 items for anxiety and 7 for depression. The scores range from 0 to 3 for each item and 0 to 21 for each subscale, with a score of 0-7 denoting no cases; $8-10$, mild cases; $11-14$, moderate cases; and $\geq 15$, severe cases.

\section{Statistical Analysis}

The categorical variables were expressed as frequencies and percentages. The continuous variables were tested if they were normally distributed, and were presented as mean \pm standard deviation or median (interquartile range, IQR) where appropriate. The $t$-test or Mann-Whitney $U$-test were used to investigate differences between groups for normally or abnormally distributed continuous variables, respectively. The Pearson's Chi-square test or Fisher's exact test was utilized to assess the differences between groups for categorical variables. The prevalence of anxiety and depression in different groups were expressed as a histogram. Multivariable logistic regression was used to investigate the effects of COPD and lung function on anxiety and depression. Confounders consisting of age, gender, marital status, employment, smoking index, cardiovascular disease, diabetes, malignant tumors and respiratory symptoms etc. were included in the multivariable-adjusted models. Model 1 was adjusted for age, gender, body mass index (BMI), marital status, education, employment, family income, smoking index, stroke, 
coronary heart disease, hypertension, diabetes, and malignancy. Model 2 was further adjusted for respiratory symptoms. Data analysis was performed using SAS version 9.4 (SAS Institute, Inc, Cary, NC, USA). Statistical significance was set at $\mathrm{P}<0.05$.

\section{Results}

In the final $\mathrm{CPH}$ study database including 50,991 participants, 1938 (3.8\%) of them did not have an intact HADS questionnaire or information about marital status, education, employment, and family income; hence, they were excluded from the final analysis in this study. Finally, 49,053 participants (20,661 men and 28,392 women) were included in the analysis. The characteristics of participants with anxiety and depression are shown in Table 1. A total of $4648(9.48 \%)$ participants had anxiety, 5528 (11.27\%) had depression, and 2946 (6.01\%) had anxiety and depression concomitantly. In the single factor analysis, anxiety was associated with older age, female sex, lower BMI, lower education level, current employment, lower family income, decreased smoking index, and more respiratory symptoms. The comorbidity profile was different between participants with anxiety and those with depression. Coronary heart disease and stroke were more common in participants with anxiety or depression; malignancy was more common in patients with anxiety. However, hypertension and diabetes were more common in patients with depression. Lung function measurements were lower in terms of $\mathrm{FEV}_{1}$ and $\mathrm{FEV}_{1} / \mathrm{FVC}$, but this trend was not observed in $\mathrm{FEV}_{1} \%$ pred. Absolute numbers and prevalence of anxiety and depression were shown in the Supplemental Table 1. The characteristics of patients with COPD with and without anxiety and depression were shown in the Supplemental Table 2.

The prevalence of anxiety and depression in patients with COPD was higher than that in non-COPD participants (Figure 1). Of the patients with COPD, 10.79\% had anxiety $(8.20 \%$ mild anxiety, $2.36 \%$ moderate anxiety, and $0.23 \%$ severe anxiety) and $13.65 \%$ had depression $(9.07 \%$ mild depression, $4.27 \%$ moderate depression, and $0.31 \%$ severe depression). Most cases of anxiety and depression were mild or moderate. In different stages of COPD, patients had an increased risk of anxiety and depression (Figure 2). In the GOLD III-IV group, 17.19\% (12.76\% mild anxiety, $4.17 \%$ moderate anxiety, and $0.26 \%$ severe anxiety) had anxiety ( $8.20 \%$ mild anxiety, $2.36 \%$ moderate anxiety, and $0.23 \%$ severe anxiety) and $21.87 \%$ (14.84\% mild depression, $6.51 \%$ moderate depression, and $0.52 \%$ severe depression) had depression. When considering the coincidence of anxiety and depression, as shown in Figure 3,7.08\% of COPD patients (especially, patients with severe disease) had anxiety and depression concomitantly. In the GOLD I, GOLD II, and GOLD III-V groups, $5.98 \%, 7.91 \%$, and $10.94 \%$, respectively, had anxiety and depression concomitantly.

COPD increased the risk of anxiety (OR 1.184, $\mathrm{P}=$ 0.001 ) in the crude model analysis (Table 2). After adjusting in models 1 and 2, COPD had no effect on anxiety. Patients in the GOLD II group did not have an increased risk of anxiety, whereas patients in the GOLD III-IV group had a higher risk of anxiety in the crude model and model 1 , but not in model 2. Similar results were found in the multivariate analysis of depression (Table 2). Patients in the GOLD III-IV group still had a higher risk of depression after adjusting for respiratory symptoms.

When comparing participants with anxiety or depression with participants with no anxiety or depression, COPD did not increase the risk of anxiety or depression after adjustment (Table 3). Patients in the GOLD III-IV COPD group had an increased risk of anxiety or depression in the crude and adjusted models.

When comparing participants with anxiety as well as depression with participants with no anxiety or depression, COPD did not increase the risk of coincidence in both models (Table 3). Patients in the GOLD II and GOLD IIIIV groups had a high risk of coincidence of anxiety and depression in the crude model and in model 1. The risk was not significant after adjusting for respiratory symptoms.

\section{Discussion}

The CPH study is the largest national pulmonary health survey in China, using a multistage stratified cluster sampling procedure in 10 provinces across the country to evaluate the disease burden of common respiratory diseases, including COPD and asthma. In addition to investigating the prevalence and risk factors of respiratory diseases, comorbidities, including anxiety and depression, were assessed using a standard questionnaire. In this study, the prevalence of anxiety was $10.79 \%$, while that of depression was $13.65 \%$ in patients with COPD. In terms of coincidence, $7.08 \%$ of patients with COPD had concomitant anxiety and depression. When adjusted for factors that may influence mental health, including age, sex, marital status, education, income, and common comorbidities, 


\begin{tabular}{|c|c|c|c|c|c|c|c|c|c|c|c|c|}
\hline a & $\begin{array}{ll}\bar{\delta} & \overline{0} \\
0 & 0 \\
\mathrm{v} & \mathrm{v}\end{array}$ & $\begin{array}{ll}\bar{o} & \bar{o} \\
0 & 0 \\
\mathrm{v} & \mathrm{v}\end{array}$ & $\begin{array}{l}\bar{o} \\
0 \\
\text { v }\end{array}$ & 员 & $\begin{array}{l}\overline{8} \\
0 \\
\mathrm{v}\end{array}$ & $\begin{array}{l}\overline{8} \\
0 \\
\text { v }\end{array}$ & $\begin{array}{l}\overline{8} \\
\dot{0} \\
\mathrm{v}\end{array}$ & & $\begin{array}{l}\overline{8} \\
\vdots \\
\text { v }\end{array}$ & $\begin{array}{l}\overline{8} \\
0 \\
v\end{array}$ & $\begin{array}{lll}\bar{\delta} & \overline{8} & \\
0 & 0 & 0 \\
& 0 & 0 \\
v & v & 0\end{array}$ & $\begin{array}{llll}\bar{\delta} & \bar{\delta} & \overline{8} & \overline{8} \\
\circ & 0 & 0 \\
\mathrm{o} & 0 \\
\mathrm{v} & \mathrm{v} & \mathrm{v} & \mathrm{v}\end{array}$ \\
\hline 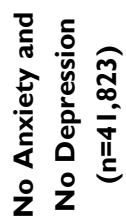 & 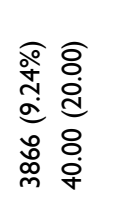 & 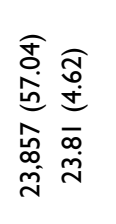 & 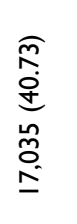 & 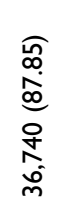 & 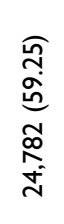 & 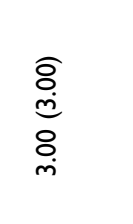 & & 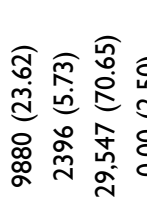 & & 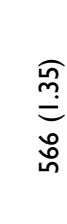 & 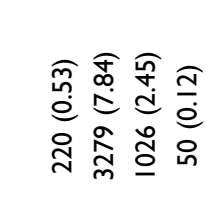 & 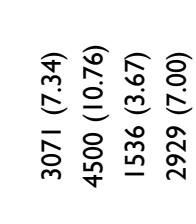 \\
\hline 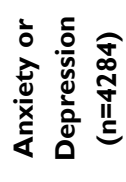 & 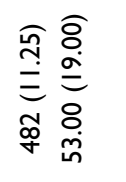 & 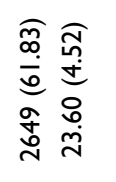 & 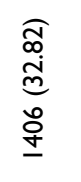 & 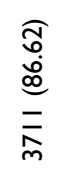 & 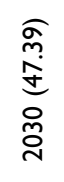 & $\begin{array}{l}\overparen{8} \\
\dot{ \pm} \\
8 \\
\dot{m}\end{array}$ & & 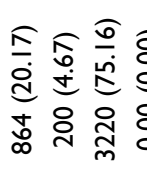 & & 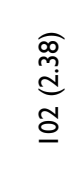 & 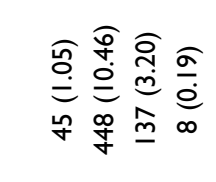 & 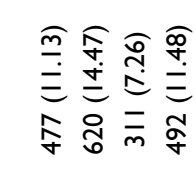 \\
\hline 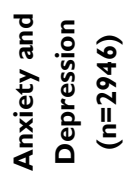 & 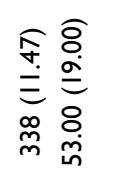 & 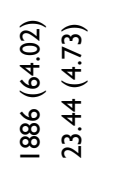 & $\begin{array}{l}\text { ָ̦ } \\
\text { ป̀ } \\
\widetilde{\infty}\end{array}$ & 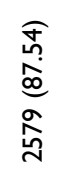 & 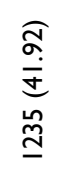 & 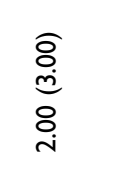 & & 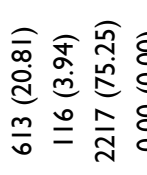 & & $\begin{array}{l}\text { } \\
\text { di } \\
\infty\end{array}$ & 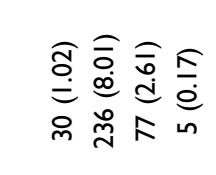 & 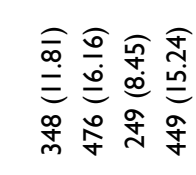 \\
\hline a & $\begin{array}{ll}\bar{\delta} & \overline{0} \\
0 & 0 \\
\mathrm{v} & \mathrm{v}\end{array}$ & $\begin{array}{ll}\bar{\delta} & \frac{m}{0} \\
\dot{0} & 0 \\
\mathrm{v}\end{array}$ & $\begin{array}{l}\bar{o} \\
\dot{0} \\
v\end{array}$ & $\stackrel{\substack{m \\
0}}{0}$ & $\begin{array}{l}\overline{8} \\
0 \\
\mathrm{v}\end{array}$ & $\begin{array}{l}\overline{8} \\
\dot{0} \\
\mathrm{v}\end{array}$ & $\begin{array}{l}\overline{8} \\
0 \\
\mathrm{v}\end{array}$ & & $\begin{array}{l}\overline{8} \\
0 \\
\text { v }\end{array}$ & $\begin{array}{l}\overline{0} \\
0 \\
\text { v }\end{array}$ & $\begin{array}{llll}\bar{\delta} & \overline{8} & 0 & \infty \\
0 & 0 & 0 \\
& 0 & 0 \\
\text { v } & \text { v } & 0 & 0\end{array}$ & $\begin{array}{llll}\bar{\delta} & \bar{o} & \overline{8} & \overline{8} \\
0 & 0 & 0 \\
v & v & v & 0 \\
v & v & v\end{array}$ \\
\hline 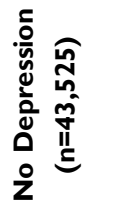 & 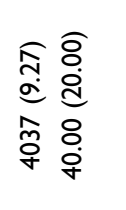 & 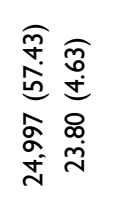 & 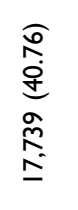 & 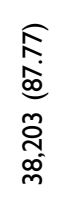 & 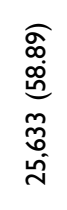 & $\begin{array}{l}\widehat{\sigma} \\
\ddot{n} \\
8 \\
\dot{m}\end{array}$ & & 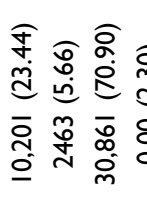 & & 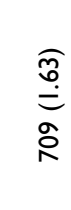 & 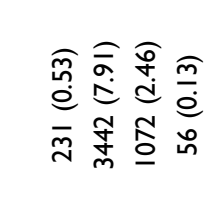 & 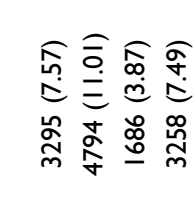 \\
\hline 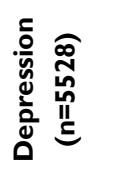 & 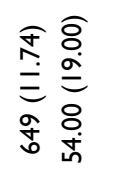 & 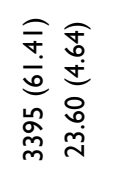 & 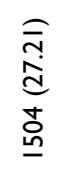 & 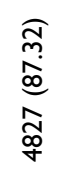 & 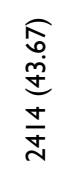 & $\begin{array}{l}\overparen{ఠ} \\
\dot{+} \\
\stackrel{\leftrightarrow}{0} \\
i\end{array}$ & & 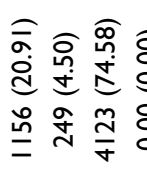 & & $\begin{array}{l}\stackrel{0}{\underline{n}} \\
\text { d. } \\
\text { m }\end{array}$ & 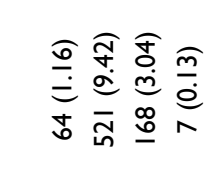 & 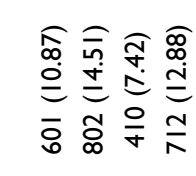 \\
\hline a & $\bar{o} \underset{0}{\overline{0}}$ & $\begin{array}{ll}\bar{\delta} & \overline{8} \\
\dot{0} & 0 \\
\mathrm{v} & \mathrm{v}\end{array}$ & $\begin{array}{l}\overline{8} \\
\dot{0} \\
\mathrm{v}\end{array}$ & ồ & $\begin{array}{l}\overline{8} \\
\dot{0} \\
\mathrm{v}\end{array}$ & $\begin{array}{l}\overline{8} \\
\dot{0} \\
v\end{array}$ & $\begin{array}{l}\overline{8} \\
\dot{0} \\
\mathrm{v}\end{array}$ & & i & $\begin{array}{l}\bar{\delta} \\
\dot{0} \\
v\end{array}$ & 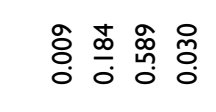 & 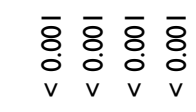 \\
\hline 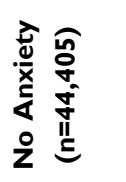 & 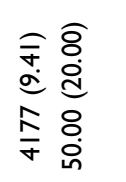 & 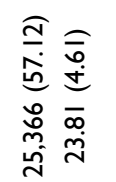 & 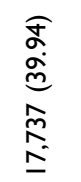 & 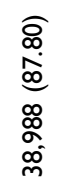 & 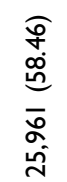 & 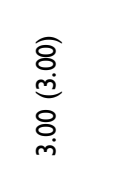 & & 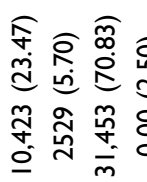 & & 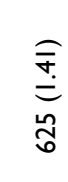 & 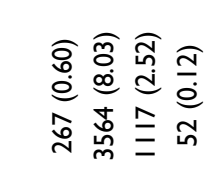 & 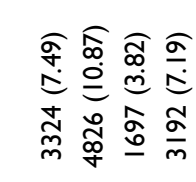 \\
\hline \multirow[t]{2}{*}{ 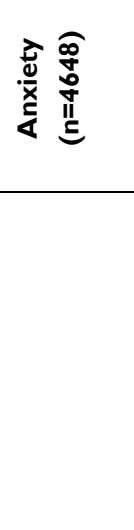 } & 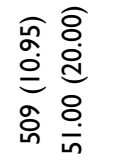 & 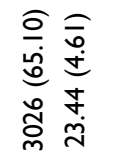 & 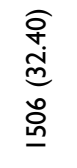 & 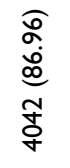 & 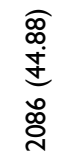 & $\begin{array}{l}\overparen{ఠ} \\
\dot{+} \\
\stackrel{\leftrightarrow}{i}\end{array}$ & & 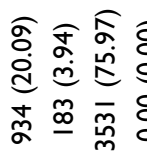 & & 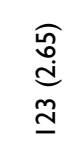 & 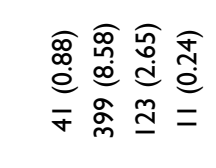 & 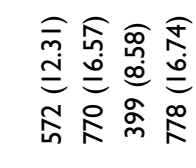 \\
\hline & 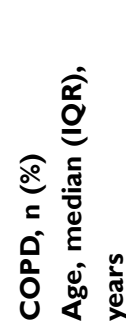 & 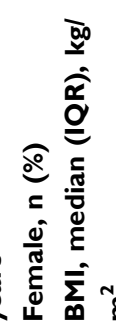 & 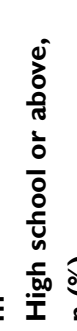 & 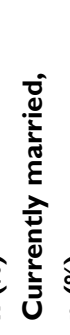 & 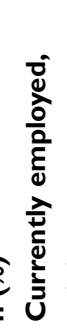 & 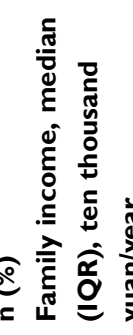 & 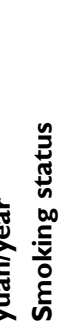 & 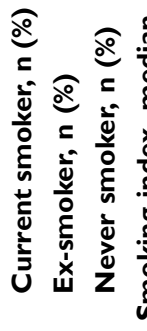 & 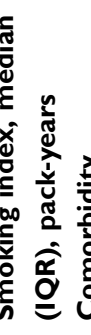 & \multicolumn{2}{|c|}{ 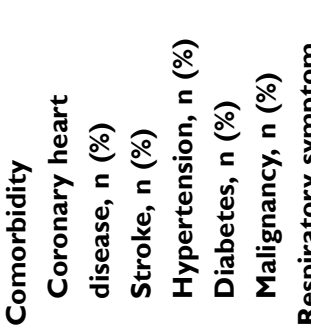 } & 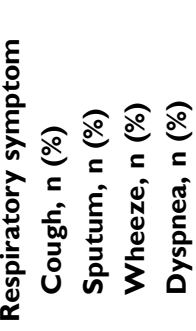 \\
\hline
\end{tabular}




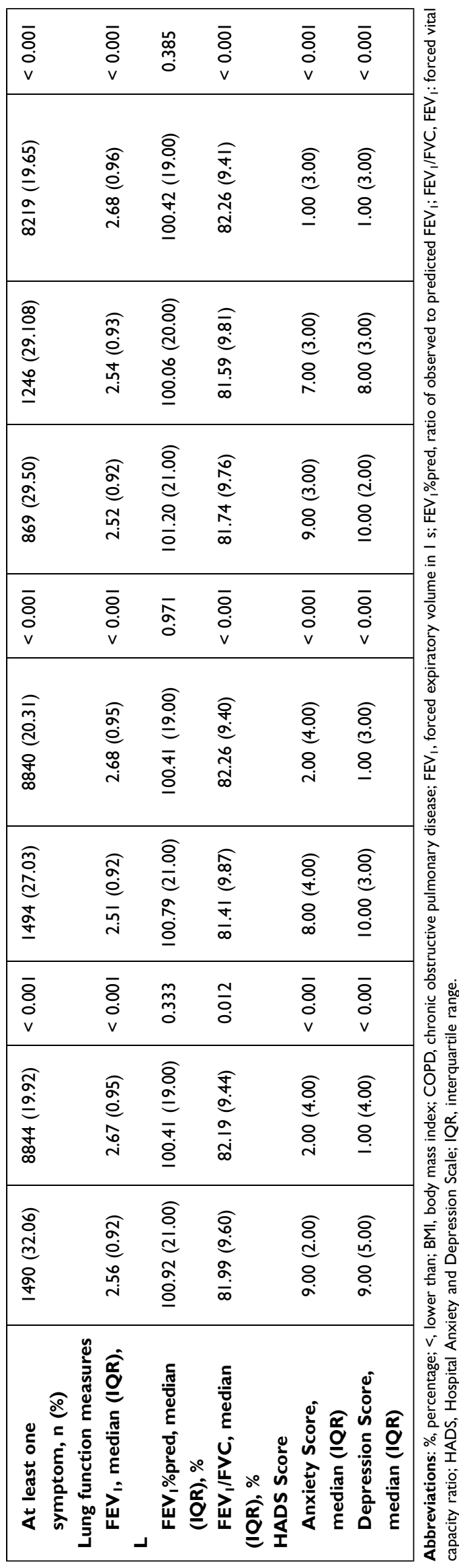

COPD did not increase the risk of anxiety or depression; however, patients with severe lung function impairment (GOLD stage III-IV) had a higher risk of anxiety, depression, and concomitant anxiety and depression. When considering respiratory symptoms, patients in the GOLD IIIIV group had a higher risk of depression or at least one of anxiety and depression. To our knowledge, this is the only study adjusting for marital status, education level, and income in the analysis of COPD and mental health status.

Among the general adult population in China, the prevalence of anxiety and depression were $5.3 \%$ and $7.2 \%$, respectively, which were assessed by the HADS questionnaire at nearly the same time as the CPH study. ${ }^{18}$ The prevalence of anxiety and depression was lower than that in previous studies conducted in COPD patients. A pooled analysis including 5552 patients with COPD and 5211 controls revealed that the prevalence of depression in COPD was $27.1 \%(25.9-28.3 \%) .{ }^{19}$ The reason for the discrepancy could be the difference in sample size, source of participants, screening tools, and COPD severity. Studies including subjects from primary care settings and tertiary care hospitals reported a higher prevalence of depression (15.2-35.7\%) because the subjects had severe lung disease. ${ }^{18,20-22}$ Another study conducted in the general population using the Center for Epidemiological Studies-Depression (CES-D) questionnaire as a screening tool reported a higher depression prevalence of $21 \%$, which may be partially explained by the fact that $43.4 \%$ of the subjects had COPD of GOLD III-IV severity. ${ }^{23}$ Liu et $\mathrm{al}^{24}$ conducted a survey in hospitals in different areas of China using HADS as the screening tool and found that the anxiety prevalence was $8.8 \%$ and depression prevalence was $15.6 \%$, and they used moderate to severe symptoms to define anxiety and depression. Willgoss et $\mathrm{al}^{8}$ investigated anxiety disorders in COPD patients in a systemic review including 10 studies and found that the prevalence of clinical anxiety ranged from $10-55 \%$ among inpatients and $13-46 \%$ among outpatients with COPD. Although the included studies had small sample sizes, they reported the prevalence of specific anxiety disorders, including generalized anxiety disorder, panic disorder, specific phobia, and social phobia. Specific depressive disorders and other anxiety disorders, including substance-induced anxiety disorder and obsessive compulsive disorder, which are common in the general population, ${ }^{5}$ have not been fully investigated in COPD patients. 


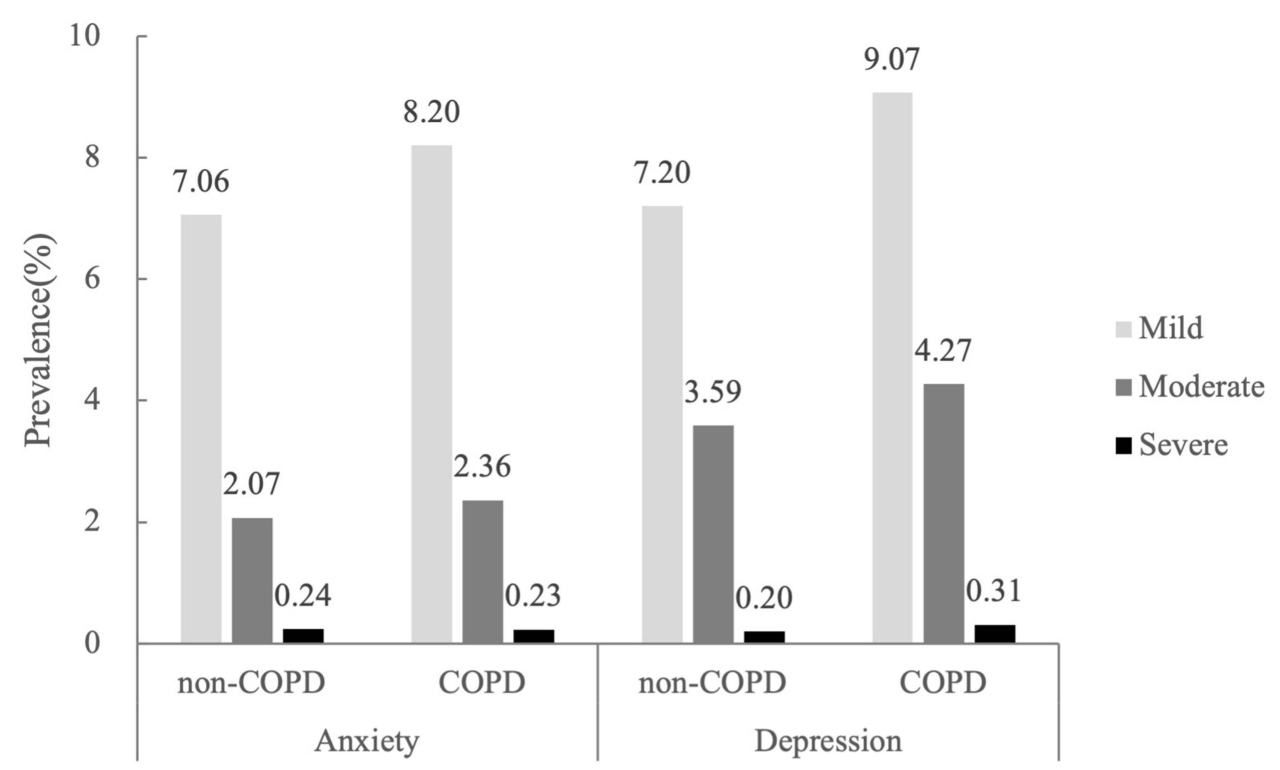

Figure I Prevalence of anxiety and depression in populations with COPD and non-COPD. The HADS subscale scores were used to define the severity of anxiety or depression, with a score of 8-10 denoting mild case, II-14 moderate case and 15 or higher severe case.

Abbreviation: COPD, chronic obstructive pulmonary disease.

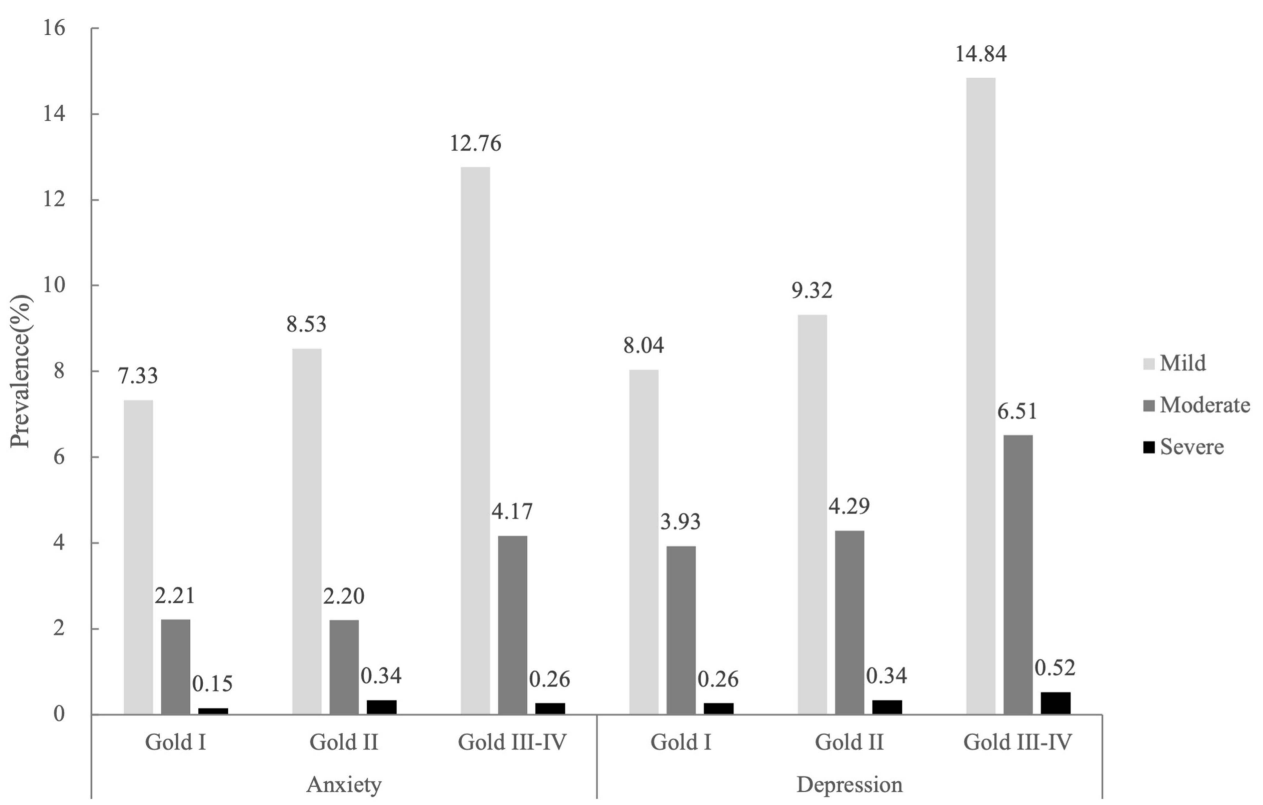

Figure 2 Prevalence of anxiety and depression in patients with different stages of COPD. The HADS subscale scores were used to define the severity of anxiety or depression, with a score of 8-10 denoting mild case, $11-14$ moderate case and 15 or higher severe case.

Abbreviations: COPD, chronic obstructive pulmonary disease; GOLD, Global Initiative for Chronic Obstructive Lung Disease.

The use of different screening instruments is an important factor, among others, that might have caused the heterogeneity across results in the included studies. Apart from the HADS questionnaire used in our study, there are a variety of self-reported questionnaires, including GDS, ${ }^{25}$ SDS (Zung), ${ }^{21}$ and CES-D. ${ }^{20,23,26}$ Although widely used, these questionnaires have not been fully validated in patients with COPD, and have not been compared to the DSM-SCID ${ }^{5}$ used in structured interviews by psychiatrists.

The factors contributing to the development of anxiety and depression in COPD have not been fully elucidated. It is plausible to assume that patients with COPD and severe 


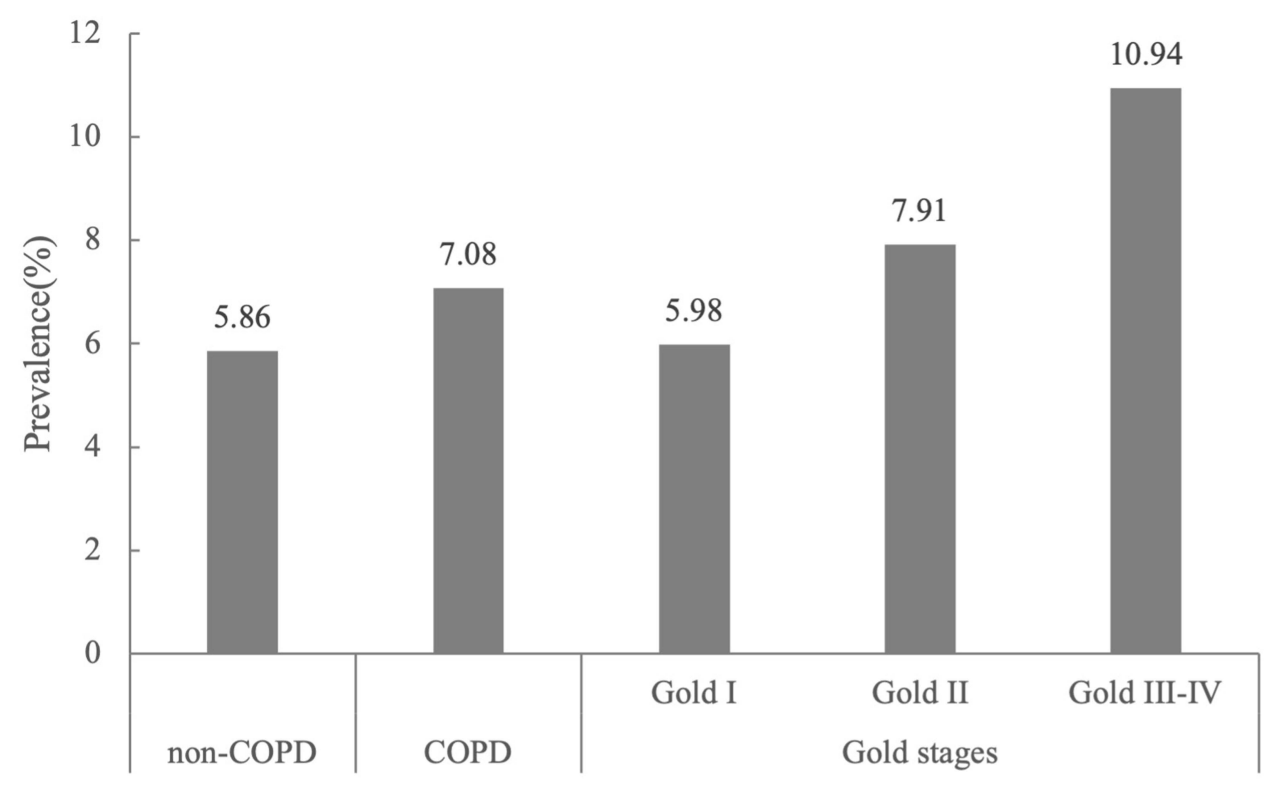

Figure 3 Coincidence of anxiety and depression in populations with COPD and non-COPD. This figure show the prevalence of subjects suffered from anxiety and depression concomitantly in COPD and non-COPD groups, as well as different GOLD stage groups.

Abbreviations: COPD, chronic obstructive pulmonary disease; GOLD, Global Initiative for Chronic Obstructive Lung Disease.

lung function impairment may have a higher risk of anxiety and depression because they may have higher smoking exposure, more respiratory symptoms, and more comorbidities. However, the effect of lung function on the risk of anxiety and depression is controversial. A previous study including 118 patients with COPD found that there was no difference in generalized psychological distress as well as moderate to severe depression between patients in the GOLD I-II group and those in the GOLD III-IV group. ${ }^{13}$ Tetikkurt et $\mathrm{al}^{27}$ found that higher GOLD stages

Table 2 Multivariate Logistic Regression for Effect of COPD and Lung Function on Anxiety or Depression

\begin{tabular}{|c|c|c|c|c|c|c|}
\hline & \multicolumn{2}{|c|}{ Crude Model } & \multicolumn{2}{|c|}{ Multivariate-Adjusted Model I* } & \multicolumn{2}{|c|}{ Multivariate-Adjusted Model $2 \ddagger$} \\
\hline & OR (95\% Cl) & $P$ value & OR $(95 \% \mathrm{CI})$ & P value & OR $(95 \% \mathrm{Cl})$ & P value \\
\hline \multicolumn{7}{|c|}{ Anxiety vs no Anxiety } \\
\hline \multicolumn{7}{|l|}{ COPD } \\
\hline No & I & & $\mathrm{I}$ & & $\mathrm{I}$ & \\
\hline Yes & I.I $84(1.074,1.306)$ & 0.001 & I.I07 $(0.994,1.232)$ & 0.064 & $0.906(0.610,1.479)$ & 0.086 \\
\hline \multicolumn{7}{|l|}{ COPD grade } \\
\hline Gold I & I & & $\mathrm{I}$ & & 1 & \\
\hline Gold II & I.I $54(0.946, I .408)$ & 0.157 & I.I85 $(0.962,1.459)$ & 0.111 & $1.050(0.848, \mathrm{I} .300)$ & 0.656 \\
\hline Gold III-IV & $2.02 I(1.503 .2 .7 \mid 7)$ & $<0.001$ & $1.925(1.391,2.665)$ & $<0.001$ & 1.361 (0.962 (1.925) & 0.082 \\
\hline \multicolumn{7}{|c|}{ Depression vs no Depression } \\
\hline \multicolumn{7}{|l|}{ COPD } \\
\hline No & I & & I & & 1 & \\
\hline Yes & $1.301(1.191,1.421)$ & $<0.001$ & $1.009(0.916,1.11 \mathrm{I})$ & 0.856 & $0.899(0.814,0.993)$ & 0.037 \\
\hline \multicolumn{7}{|l|}{ COPD grade } \\
\hline Gold I & I & & I & & I & \\
\hline Gold II & I.I58 $(0.968,1.385)$ & 0.108 & I.I57 $(0.96 \mathrm{I}, \mathrm{I} .393)$ & 0.125 & I.077 (0.89I,I.302) & 0.443 \\
\hline Gold III-IV & $2.007(1.530,2.633)$ & $<0.001$ & $1.744(1.296,2.347)$ & $<0.001$ & $1.412(1.031,1.932)$ & 0.031 \\
\hline
\end{tabular}

Notes: *Model I: adjusted age, sex, BMI, marriagement, education, employment, family income, smoking index, stroke, coronary heart disease, hypertension, diabetes and malignancy. ‡Model 2: adjusted model I+ respiratory symptoms (cough, sputum, wheeze, dyspnea).

Abbreviations: <, lower than; $95 \% \mathrm{Cl}$, 95\% Confidence Interval; COPD, chronic obstructive pulmonary disease; GOLD, Global Initiative for Chronic Obstructive Lung Disease. 
Table 3 Multivariate Logistic Regression for Effect of COPD and Lung Function on Anxiety and Depression

\begin{tabular}{|c|c|c|c|c|c|c|}
\hline & \multicolumn{2}{|c|}{ Crude Model } & \multicolumn{2}{|c|}{$\begin{array}{l}\text { Multivariate-Adjusted } \\
\text { Model I* }\end{array}$} & \multicolumn{2}{|c|}{$\begin{array}{l}\text { Multivariate-Adjusted } \\
\text { Model } 2 \ddagger\end{array}$} \\
\hline & OR $(95 \% \mathrm{CI})$ & $P$ value & OR $(95 \% \mathrm{CI})$ & $P$ value & OR $(95 \% \mathrm{CI})$ & $P$ value \\
\hline \multicolumn{7}{|c|}{ Anxiety or Depression vs no } \\
\hline \multicolumn{7}{|c|}{ Anxiety and no Depression } \\
\hline \multicolumn{7}{|c|}{ COPD } \\
\hline No & 1 & & 1 & & 1 & \\
\hline Yes & $1.245(1.126,1.376)$ & $<0.001$ & $1.069(0.96 \mathrm{I}, \mathrm{I} .189)$ & 0.218 & $0.931(0.834,1.039)$ & 0.201 \\
\hline \multicolumn{7}{|l|}{ COPD grade } \\
\hline Gold I & I & & 1 & & 1 & \\
\hline Gold II & $0.963(0.782,1.186)$ & 0.723 & $0.96 \mathrm{I}(0.779, \mathrm{I} .180)$ & 0.714 & $0.870(0.701,1.079)$ & 0.205 \\
\hline Gold III-IV & $2.067(1.528,2.796)$ & $<0.001$ & $1.996(1.464,2.720)$ & $<0.001$ & $1.502(1.078,2.093)$ & 0.016 \\
\hline \multicolumn{7}{|c|}{ Coincidence of Anxiety and } \\
\hline \multicolumn{7}{|c|}{ Depression vs no Anxiety and no } \\
\hline \multicolumn{7}{|l|}{ Depression } \\
\hline \multicolumn{7}{|l|}{ COPD } \\
\hline No & $\mathrm{I}$ & & 1 & & 1 & \\
\hline Yes & $1.272(1.131,1.432)$ & $<0.001$ & $1.062(0.932,1.209)$ & 0.368 & $0.887(0.775,1.015)$ & 0.08 \\
\hline \multicolumn{7}{|l|}{ COPD grade } \\
\hline Gold I & 1 & & I & & 1 & \\
\hline Gold II & $1.308(1.030,1.658)$ & 0.028 & $1.331(1.038,1.706)$ & 0.024 & $1.200(0.930,1.547)$ & 0.16 \\
\hline Gold III-IV & $2.179(1.515,3.133)$ & $<0.001$ & $1.978(1.328,2.948)$ & 0.001 & I.477 $(0.968,2.252)$ & 0.07 \\
\hline
\end{tabular}

Notes: *Model I: adjusted age, sex, BMI, marriagement, education, employment, family income, smoking index, stroke, coronary heart disease, hypertension, diabetes and malignancy. ¥Model 2: adjusted model I+ respiratory symptoms (cough, sputum, wheeze, dyspnea).

Abbreviations: <, lower than; $95 \% \mathrm{Cl}$, 95\% confidence Interval; COPD, chronic obstructive pulmonary disease; GOLD, Global Initiative for Chronic Obstructive Lung Disease.

were associated with anxiety and depression, while $\mathrm{FEV}_{1} \%$ pred was not significantly associated with HADS score. They also found that dyspnea and reduced exercise capacity were significantly related to anxiety and depression symptoms. These findings suggest that lung function cannot fully explain the mental status of patients with COPD. Respiratory symptoms and other factors have a psychological impact on patients with COPD. In our study, patients in the GOLD III-IV group did not have an increased risk of anxiety or concomitant anxiety and depression after adjusting for respiratory symptoms and other factors. However, the risk of depression significantly increased even after adjustment, suggesting that there is an intrinsic association between depression and impairment of lung function.

The exact mechanisms linking anxiety and depression with COPD remain unclear; however, the relationship between them is partially explained by confounding factors, such as smoking, exercise capacity, and disease severity. There is evidence suggesting that chronic inflammatory factors, such interleukin-6 and C-reactive protein, may be associated with depressive symptoms in patients with COPD. ${ }^{28,29}$

It is important to recognize anxiety and depression in patients with COPD. Emerging evidence suggests that pulmonary rehabilitation and mind-body exercise can improve the symptoms of anxiety and depression. ${ }^{30,31}$ The benefits of psychological intervention for anxiety and depression in patients with COPD in terms of healthcare resource use and effective self-management of COPD have been evaluated in randomized controlled trials. ${ }^{32}$

Although this is the largest study assessing the prevalence of anxiety and depression in Chinese patients with COPD, it has several limitations. First, although the HADS questionnaire has been widely used as a screening tool in other studies, it did not provide a reliable clinical diagnosis of anxiety or depressive disorders. Second, possible confounders, such as previous diagnosis of anxiety and depression, medications, exercise measurements, and inflammatory factors, were not evaluated in our study. Third, we could not assess any possible causal links between lung function and depression because of the cross-sectional nature of the study. 


\section{Conclusion}

Our study found that anxiety and depression are prevalent in patients with COPD in China. Severe lung function impairment was independently associated with a significantly higher risk of depression. Psychological interventions for anxiety and depression should be evaluated in future studies.

\section{Abbreviation}

BMI, Body Mass index; CES-D, Center for Epidemiological Studies-Depression; COPD, Chronic Obstructive Pulmonary Disease; CPH, China Pulmonary Health; FEV1, Forced Expiratory Volume in $1 \mathrm{~s}$; FEV1\%pred, ratio of observed to predicted FEV1; FVC, Forced Vital Capacity; GOLD, Global Initiative for Chronic Obstructive Lung Disease; HADS, Hospital Anxiety and Depression Scale.

\section{Summary}

We analyzed data of 49,053 participants to assess the prevalence of anxiety and depression in COPD patients and their relationship with GOLD stages. We found $10.79 \%$ of the patients with COPD had anxiety, $13.65 \%$ had depression, and $7.08 \%$ had anxiety and depression concomitantly. After adjusting confounders, the lung function impairment in the GOLD III-IV group was significantly associated with depression.

\section{Acknowledgments}

This research was supported by National Key R\&D Program of China (grant numbers 2018YFC1315102 and 2016YFC1303900).

\section{Disclosure}

The authors report no conflicts of interest in this work.

\section{References}

1. Wang C, Xu J, Yang L, et al. Prevalence and risk factors of chronic obstructive pulmonary disease in China (the China Pulmonary Health [CPH] study): a national cross-sectional study. Lancet. 2018;391 (10131):1706-1717. doi:10.1016/S0140-6736(18)30841-9

2. Zhou M, Wang H, Zeng X, et al. Mortality, morbidity, and risk factors in China and its provinces, 1990-2017: a systematic analysis for the Global Burden of Disease Study 2017. Lancet. 2019;394 (10204):1145-1158. doi:10.1016/S0140-6736(19)30427-1

3. Negewo NA, Gibson PG, McDonald VM. COPD and its comorbidities: impact, measurement and mechanisms. Respirology. 2015;20 (8):1160-1171. doi:10.1111/resp. 12642

4. Miller J, Edwards LD, Agusti A, et al. Comorbidity, systemic inflammation and outcomes in the ECLIPSE cohort. Respir Med. 2013;107 (9):1376-1384. doi:10.1016/j.rmed.2013.05.001
5. Huang Y, Wang Y, Wang H, et al. Prevalence of mental disorders in China: a cross-sectional epidemiological study. Lancet Psychiatry. 2019;6(3):211-224. doi:10.1016/S2215-0366(18)30511-X

6. Zhang MW, Ho RC, Cheung MW, Fu E, Mak A. Prevalence of depressive symptoms in patients with chronic obstructive pulmonary disease: a systematic review, meta-analysis and meta-regression. Gen Hosp Psychiatry. 2011;33(3):217-223. doi:10.1016/j.genhospps ych.2011.03.009

7. Kunik ME, Roundy K, Veazey C, et al. Surprisingly high prevalence of anxiety and depression in chronic breathing disorders. Chest. 2005;127(4):1205-1211. doi:10.1378/chest.127.4.1205

8. Willgoss TG, Yohannes AM. Anxiety disorders in patients with COPD: a systematic review. Respir Care. 2013;58(5):858-866. doi: $10.4187 /$ respcare. 01862

9. Griffith MF, Chen HP, Bekelman DB, et al. Comorbid anxiety and depression, though underdiagnosed, are not associated with high rates of low-value care in patients with COPD. Ann Am Thorac Soc. 2020. doi:10.1513/AnnalsATS.201911-854OC

10. Panagioti M, Scott C, Blakemore A, Coventry PA. Overview of the prevalence, impact, and management of depression and anxiety in chronic obstructive pulmonary disease. Int J Chron Obstruct Pulmon Dis. 2014;9:1289-1306. doi:10.2147/COPD.S72073

11. Vikjord SAA, Brumpton BM, Mai XM, Vanfleteren L, Langhammer A. The association of anxiety and depression with mortality in a COPD cohort. The HUNT study, Norway. Respir Med. 2020;171:106089. doi:10.1016/j.rmed.2020.106089

12. Xu W, Collet JP, Shapiro S, et al. Independent effect of depression and anxiety on chronic obstructive pulmonary disease exacerbations and hospitalizations. Am J Respir Crit Care Med. 2008;178 (9):913-920. doi:10.1164/rccm.200804-6190C

13. Wagena EJ, Arrindell WA, Wouters EF, van Schayck CP. Are patients with COPD psychologically distressed? Eur Respir J. 2005;26 (2):242-248. doi:10.1183/09031936.05.00010604

14. Huang K, Yang T, Xu J, et al. Prevalence, risk factors, and management of asthma in China: a national cross-sectional study. Lancet. 2019;394(10196):407-418. doi:10.1016/S0140-6736(19)31147-X

15. Xiao D, Chen Z, Wu S, et al. Prevalence and risk factors of small airway dysfunction, and association with smoking, in China: findings from a national cross-sectional study. Lancet Respir Med. 2020;8 (11):1081-1093. doi:10.1016/S2213-2600(20)30155-7

16. Zigmond AS, Snaith RP. The hospital anxiety and depression scale. Acta Psychiatr Scand. 1983;67(6):361-370. doi:10.1111/j.16000447.1983.tb09716.x

17. Lam CL, Pan PC, Chan AW, Chan SY, Munro C. Can the Hospital Anxiety and Depression (HAD) scale be used on Chinese elderly in general practice? Fam Pract. 1995;12(2):149-154. doi:10.1093/fam$\mathrm{pra} / 12.2 .149$

18. Lou P, Zhu Y, Chen P, et al. Prevalence and correlations with depression, anxiety, and other features in outpatients with chronic obstructive pulmonary disease in China: a cross-sectional case control study. BMC Pulm Med. 2012;12:53. doi:10.1186/1471-2466-12-53

19. Matte DL, Pizzichini MM, Hoepers AT, et al. Prevalence of depression in COPD: a systematic review and meta-analysis of controlled studies. Respir Med. 2016;117:154-161. doi:10.1016/j. rmed.2016.06.006

20. Van Manen JG, Bindels PJ, Dekker FW, IJzermans CJ, Van der Zee JS, Schade E. Risk of depression in patients with chronic obstructive pulmonary disease and its determinants. Thorax. 2002;57(5):412-416. doi:10.1136/thorax.57.5.412

21. Di Marco F, Verga M, Reggente M, et al. Anxiety and depression in COPD patients: the roles of gender and disease severity. Respir Med. 2006;100(10):1767-1774. doi:10.1016/j.rmed.2006.01.026

22. Wong TS, Xiang YT, Tsoh J, et al. Depressive disorders in older patients with chronic obstructive pulmonary disease (COPD) in Hong Kong: a controlled study. Aging Ment Health. 2014;18 (5):588-592. doi:10.1080/13607863.2013.856862 
23. Persson LJ, Aanerud M, Hiemstra PS, Hardie JA, Bakke PS, Eagan TM. Chronic obstructive pulmonary disease is associated with low levels of vitamin D. PLoS One. 2012;7(6):e38934. doi:10.1371/journal.pone.0038934

24. Liu Y, Tian X, Guo X, et al. Prevalence of anxiety and depression in chronic obstructive pulmonary disease. Chin J Respir Crit Care Med. 2020;19(5):425-429.

25. Omachi TA, Katz PP, Yelin EH, et al. Depression and health-related quality of life in chronic obstructive pulmonary disease. Am J Med. 2009;122(8):778 e9-15. doi:10.1016/j.amjmed.2009.01.036

26. Hanania NA, Mullerova H, Locantore NW, et al. Determinants of depression in the ECLIPSE chronic obstructive pulmonary disease cohort. Am J Respir Crit Care Med. 2011;183(5):604-611. doi:10.1164/rccm.201003-0472OC

27. Tetikkurt C, Ozdemir I, Tetikkurt S, Yilmaz N, Ertan T, Bayar N. Anxiety and depression in COPD patients and correlation with sputum and BAL cytology. Multidiscip Respir Med. 2011;6(4):226-231. doi:10.1186/2049-6958-6-4-226

28. Lu Y, Feng L, Feng L, Nyunt MS, Yap KB, Ng TP. Systemic inflammation, depression and obstructive pulmonary function: a population-based study. Respir Res. 2013;14:53. doi:10.1186/14659921-14-53
29. Yohannes AM, Alexopoulos GS. Depression and anxiety in patients with COPD. Eur Respir Rev. 2014;23(133):345-349. doi:10.1183/ 09059180.00007813

30. Gordon CS, Waller JW, Cook RM, Cavalera SL, Lim WT, Osadnik CR. Effect of pulmonary rehabilitation on symptoms of anxiety and depression in COPD: a systematic review and meta-analysis. Chest. 2019;156(1):80-91. doi:10.1016/j. chest.2019.04.009

31. Li Z, Liu S, Wang L, Smith L. Mind-body exercise for anxiety and depression in COPD patients: a systematic review and meta-analysis. Int J Environ Res Public Health. 2019;17(1). doi:10.3390/ ijerph17010022

32. Sohanpal R, Pinnock H, Steed L, et al. Tailored, psychological intervention for anxiety or depression in people with chronic obstructive pulmonary disease (COPD), TANDEM (Tailored intervention for ANxiety and DEpression Management in COPD): protocol for a randomised controlled trial. Trials. 2020;21(1):18. doi:10.1186/ s13063-019-3800-y

\section{Publish your work in this journal}

The International Journal of COPD is an international, peer-reviewed journal of therapeutics and pharmacology focusing on concise rapid reporting of clinical studies and reviews in COPD. Special focus is given to the pathophysiological processes underlying the disease, intervention programs, patient focused education, and self management protocols. This journal is indexed on PubMed Central, MedLine and CAS. The manuscript management system is completely online and includes a very quick and fair peer-review system, which is all easy to use. Visit http://www.dovepress.com/testimonials.php to read real quotes from published authors. 